\title{
O Universo Lúdico da Programação de Computadores com Logo no Ensino Fundamental
}

\author{
Aline Sousa, Sivaldo Silva, Alberto A. C. Raiol ${ }^{1}$, João Sarges ${ }^{1}$, Fábio Bezerra ${ }^{1}$ \\ ${ }^{1}$ Instituto Ciberespacial - Universidade Federal Rural da Amazônia (UFRA) \\ Av. Presidente Tancredo Neves, 2501 - Belém - PA \\ \{fabio.bezerra\}@ufra.edu.br
}

\begin{abstract}
Build a computer program requires skills as the ordination of thought, abstract thinking, problem solving and logical and mathematical reasoning. Promote contact elementary students with the world of computer programming is an opportunity to stimulate the development of these skills. This paper reports the experience that elementary school students from two public schools in Belém-PA had with computer programming. These students are from the seventh and eighth years, and they had computer programming classes with the Logo language in KTurtle environment. We observed that the students who had contact with this experience improved their performance on math, when compared with classmates that did not have contact. We hope that the reported experience stimulates the adoption of computer programming initiatives in the curriculum of elementary school students.
\end{abstract}

Resumo. Criar um programa de computador exige habilidades como a ordenação do pensamento, pensamento abstrato, resolução de problemas e raciocínio lógico e matemático. Promover o contato de alunos do ensino fundamental com o universo da programação de computadores é uma oportunidade de estimular, de forma mais acelerada, o desenvolvimento dessas habilidades. Este artigo relata a experiência que alunos do ensino fundamental de duas escolas públicas em Belém-PA tiveram com a programação de computadores. No caso, alunos do sétimo e oitavo anos assistiram aulas de Logo no ambiente KTurtle. Observamos que os alunos que tiveram contato com essa experiência melhoraram o desempenho na disciplina de matemática. Esperamos que relatos como os apresentados neste artigo estimulem a adoção da disciplina de programação de computadores na grade curricular de alunos do ensino fundamental.

\section{Introdução}

Não é recente o desejo de incluir no currículo da educação básica (fundamental ou média) conteúdo sobre ciência da computação [Gregolin 2009, Fincher et al. 2010, Pardamean et al. 2011, de França et al. 2012, Costa et al. 2012, Bezerra e Dias 2014]. Em países como Estados Unidos e Brasil, que estão posicionados entres as seis maiores economias do mundo, sabe-se que criar tecnologia é fundamental para a economia. Nesse contexto, programas e iniciativas que fortaleçam as áreas de ciência, tecnologia, engenharias e matemáticas tornam-se fundamentais, a exemplo da STEM Education Coalition ${ }^{1}$.

\footnotetext{
${ }^{1}$ http://www.stemedcoalition.org/
} 
Em [de França et al. 2012], os autores relatam a experiência do ensino de Computação na Educação Básica envolvendo alunos do nono ano do ensino fundamental. Para tanto, foi aplicada a metodologia de Computação Desplugada (Computer Science Unplugged $^{2}$ ). Um estudo que também utiliza a computação desplugada é apresentado em [Costa et al. 2012]. Ao contrário do primeiro, este experimento aplica a metodologia através de gincanas. Segundo os autores, com base em observações e anotações dos licenciandos, foi possível identificar entre os alunos aqueles que apresentaram maior facilidade na resolução de problemas lógicos, bem como assimilação do conteúdo de computação, capacidade de trabalho em equipe e organização do pensamento.

Com relação ao ensino de programação de computadores há os trabalhos de [Fincher et al. 2010, Pardamean et al. 2011, Friedrich et al. 2012, Bezerra e Dias 2014]. Em [Fincher et al. 2010] os autores comparam a eficácia de três ambientes de programação de computadores: Alice, Greenfoot e Scratch. Segundo os autores, os ambientes possuem características similares consideradas importantes para o engajamento dos aprendizes, como programação visual, aprendizado de forma lúdica, desenvolvimento de programas gráficos e interativos, rico arsenal de recursos multimídia, caso do Alice e Scratch, bem como a capacidade de desenvolvimento de aplicações mais sofisticadas, preparando os aprendizes para o uso de ambientes de programação mais gerais, por exemplo, caso do Greenfoot, que é baseado em Java. Enquanto em [Pardamean et al. 2011] os autores relatam um aumento significativo na criatividade da resolução de problemas em um experimento com 85 crianças do ensino fundamental.

Outros esforços são em direção ao suporte da computação a disciplinas da educação básica [Freitas e Santos 2005, Barcelos e Silveira 2012, Torezani et al. 2013] e até mesmo a uma educação inclusiva [Alcoba 2003]. Em [Barcelos e Silveira 2012], os autores apresentam algumas tendências de pesquisa relacionadas ao ensino de computação como ciência básica, discutindo suas possíveis relações com a educação matemática, bem como fazem um paralelo com as competências definidas para o ensino de Matemática nos Parâmetros Curriculares Nacionais para o Ensino Médio. Espera-se com isso, proporcionar alguns caminhos para que as áreas possam se beneficiar mutuamente com o desenvolvimento de estratégias pedagógicas conjuntas. Em [Freitas e Santos 2005] os autores apresentam uma experiência interessante de trabalho com a matemática com alunos da sétima série do ensino fundamental, que utiliza a linguagem Logo na construção de fractais. Nesse trabalho os autores revelam que o sucesso da experiência está relacionado ao contato com a informática que os alunos vivenciavam há tempos na escola. Enquanto que em [Torezani et al. 2013] os autores apresentam o ambiente NewProg e os resultados do estudo que fizeram com crianças de cinco a oito anos que usaram o referido ambiente.

Motivados pelo desenvolvimento do raciocínio lógico como instrumento de seleção mais crítica das informações mais corretas, dentre as tantas disponíveis na internet, os autores em [Friedrich et al. 2012] apresentam um método pedagógico de inserção da lógica de programação para crianças de sete a dez anos, usando a linguagem Logo e o projeto Lego Mindstorms.

Portanto, os diferentes estudos citados acima relatam que o contato com a

\footnotetext{
${ }^{2}$ Metodologia disponível em http://csunplugged.org/.
} 
programação de computadores, desde cedo (ensino fundamental), favorece o desenvolvimento de habilidades cognitivas como: resolução de problemas, criatividade e autoria, e o espírito crítico. Então, convergente com essa tendência de modificar o currículo da educação básica para incluir o ensino da computação, apresentamos neste trabalho uma proposta de ensino de programação de computadores com a linguagem Logo no ensino fundamental. Diferente da experiência relatada em [Bezerra e Dias 2014], este trabalho apresenta com mais profundidade o plano de ensino utilizado nas diferentes escolas e turmas, discute os efeitos que o contato com a programação de computadores poderia ter no desempenho da disciplina de matemática, além de incluir dados de mais turmas. Além disso, apresentamos nossa experiência com o ensino da programação de computadores no ensino fundamental maior (sexto ao nono ano) em duas escolas públicas estaduais. Assim, esperamos com as ações da referida proposta: (i) apresentar a área de computação como ciência, que busca por soluções de problemas de áreas diversas; (ii) desenvolver a habilidade com algoritmo desde cedo, no caso, desde o ensino fundamental; (iii) atrair novos talentos para a área de computação, que mundialmente carece de profissionais; e finalmente, (iv) melhorar o desempenho nas disciplinas de matemática e português.

Este artigo é resultado da experiência de alunos do curso de Licenciatura em Computação da Universidade A, que atuam no Programa Institucional de Bolsas de Iniciação à Docência (PIBID), que é um programa federal financiado pelo Ministério da Educação (MEC), através da Coordenação de Aperfeiçoamento de Pessoal de Nível Superior (CAPES). Esperamos que o relato da experiência, bem como os resultados apresentados aqui, sirvam de instrumento para a inclusão do ensino de programação de computadores na educação fundamental. Pata tanto, organizamos este artigo da seguinte forma: na Seção 2 apresentamos o plano de ensino de programação de computadores com a linguagem Logo; na Seção 3 apresentamos as escolas que abrigaram o projeto, o método de trabalho e pesquisa utilizado, além dos instrumentos de avaliação do projeto, cujos resultados serão apresentados na Seção 4; finalmente, na Seção 5 resumimos os resultados e limitações dos esforços iniciais deste projeto, bem como apresentamos algumas ações que planejamos para o futuro.

\section{A Oficina de Logo e o Ambiente KTurtle}

O Logo é uma linguagem de programação desenvolvida no MIT, em 1967, por Seymourt Papert e outros colaboradores, que permite que adultos e crianças, mesmo as menores, usem os computadores como uma ferramenta de aprendizagem [Papert 1993, Pardamean et al. 2011]. Ela é uma linguagem de programação criada para o ambiente escolar, com o objetivo de ensinar linguagem de programação na educação básica, por ser uma linguagem que permite a fácil compreensão dos alunos. É uma linguagem rica em recursos e não exige o domínio da matemática, facilitando o acesso dos principiantes [Gregolin 2009]. Neste trabalho adotamos o KTurtle, que é uma opção gratuita, disponível para a plataforma Linux, que permite a configuração para o português do Brasil, além de permitir o uso dos comandos da linguagem Logo em português.

Planejamos uma oficina com carga horária total de 40 horas, que foi dividida em dois estágios: o básico com 16 horas de duração, e o avançado com 24 horas de duração (ver Tabela 1). Ao completar o estágio básico o aluno seria capaz de entender o conceito de algoritmo, como construir um programa de computador usando alguns elementos sintáticos e semânticos da linguagem Logo, e a utilizar o ambiente KTurtle. Enquanto que 
Tabela 1. Conteúdo Programático: Básico e Avançado

\begin{tabular}{|c|c|}
\hline Básico & Avançado \\
\hline 1. Conceitos de computação & 1. Variáveis \\
\hline 2. Introdução ao algoritmo & Tipos de Variáveis \\
\hline 3. Numeracão binário & Concatenando Variáveis \\
\hline 4. Vocabulário Inicial & 2. Entrada e Saída \\
\hline 5. Ambiente KTurtle & 3. Operadores Matemáticos \\
\hline 6. Comandos Básicos & 4. Funções Matemáticas \\
\hline Movimentação & 5. Outros Operadores \\
\hline Rotação & Lógicos \\
\hline 7. Comandos de Apresentação & Comparação \\
\hline Cores & 6. Estruturas de Seleção \\
\hline Rastro & 7. Estruturas de Repetição \\
\hline 8. Comandos de Escrita & 8. Criando uma Função \\
\hline Cor da Fonte & \\
\hline Tamanho da Fonte & \\
\hline
\end{tabular}

ao completar o estágio avançado da oficina o aluno deveria ser capaz de utilizar com maior domínio o ambiente KTurtle, construir programas que combinam diferentes estruturas de programação (sequência, seleção e repetição), e a desenvolver suas próprias funções.

\subsection{Oficina Básica}

A oficina básica, no formato intensivo, foi planejada para ser executada em quatro dias de aula, com quatro horas de aula por dia. Os exercícios que podem ser explorados na oficina básica podem visitar conteúdos da matemática, especificamente da geometria, como ilustrada na Figura 1 (ex: figuras geométricas, ângulos, operações com ângulos, reta, etc.). A seguir, apresentamos o conteúdo para cada dia de aula.

Primeiro Dia Os alunos terão acesso ao computador apenas na última hora de aula. Neste primeiro dia eles desenvolverão habilidades como: (i) lógica; (ii) conceitos computacionais; e (iii) operações elementares do ambiente KTurtle (ex: abrir, editar e salvar um programa). O conteúdo é apresentado através de atividades lúdicas como "O mestre mandou", que apresenta a noção de algoritmo e o problema da "A ovelha, o pastor e o lobo" e "Torre de Hanói", que desenvolve o raciocínio lógico.

Segundo Dia A partir do segundo dia as aulas são sempre com atividades no computador. Aqui eles desenvolverão habilidades como: (i) pensamento computacional; (ii) operação do ambiente KTurtle; (iii) linguagem de programação; e (iv) linguagem Logo. O conteúdo é apresentado de forma prática, com bastante exercícios e exemplos de código.

Terceiro Dia As atividades e recursos apresentados neste dia são importantes para manter os alunos motivados e estimulá-los a criar desenhos mais sofisticados e coloridos. 


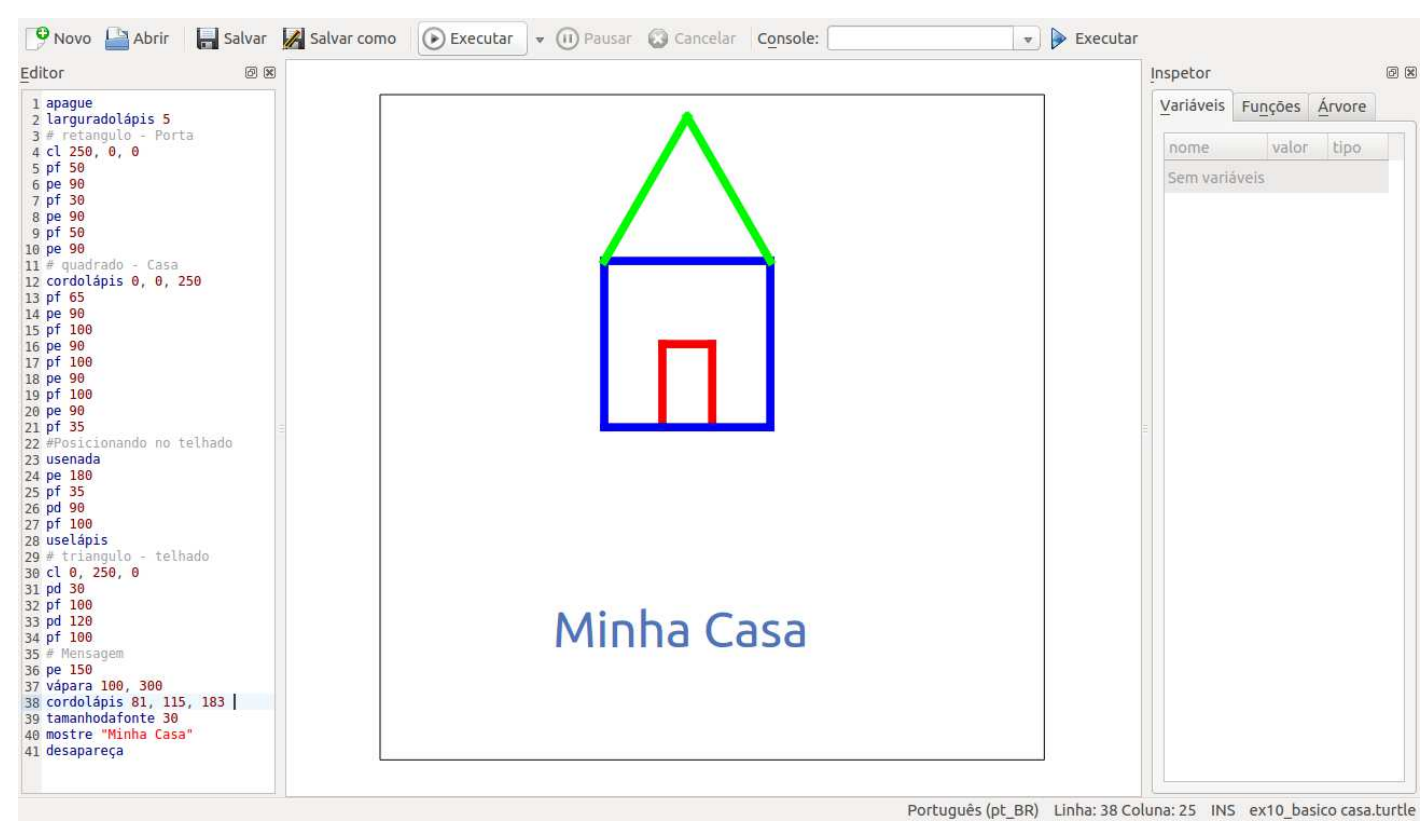

Figura 1. Exemplo de Exercício da Oficina Básica

Quarto Dia Finalmente, no último dia de aula do plano básico, os alunos são expostos a mais desafios e a utilizar a área de desenho do KTurtle para apresentar mensagens em diferentes tamanhos, estilos e cores.

Uma alternativa ao formato intensivo de quatro dias é o formato fracionado, com um intervalo espaçado na semana, ideal para a inclusão do conteúdo no currículo do ensino básico, assemelhando-se a presença de outras disciplinas, como português e história. No caso, são necessárias oito aulas, de duas hora cada, duas vezes na semana.

\subsection{Oficina Avançada}

A oficina avançada, no formato intensivo, foi planejada para ser executada em seis dias de aula, com quatro horas de aula por dia. Os exercícios que podem ser explorados na oficina avançada podem visitar conteúdos da matemática, especificamente sobre funções e álgebra (ex: expressões matemáticas, operações matemáticas, funções, etc.).

$\mathrm{Na}$ oficina avançada, todas as aulas são totalmente práticas e os desafios exigem um pensamento computacional mais apurado dos alunos. Por essa razão, a oficina avançada tem como pré-requisito o conteúdo apresentado na oficina básica. A seguir, apresentamos o conteúdo que deve ser explorado em cada dia de aula.

Primeiro Dia Os alunos desenvolverão habilidades como: (i) variável; (ii) tipo (ou domínio de valores) de uma variável; e (iii) atribuição de valores e uso de uma variável.

Segundo Dia As operações de entrada e saída são fundamentais em um sistema computacional. Por exemplo, porque já possuem o conceito de variável e desenvolverão a habilidade de ler uma entrada de dados, eles poderão refazer os exercícios e desafios da oficina básica, mas com um resultado (saída) que depende da entrada informada ao programa.

Terceiro Dia Aqui os alunos terão contato com expressões diferentes do que estão acostumados a ver, pois trabalharão com expressões e operadores lógicos. 
Quarto Dia Aqui os alunos desenvolverão programas que tomam decisões, pois desenvolverão a habilidade de trabalhar com estruturas como: "se idade maior do que 12 anos então paga inteira, senão paga meia".

Quinta Dia Aqui os alunos desenvolverão habilidades para trabalhar com a repetição de comandos de forma mais enxuta, ou seja, trechos repetidos podem ser reduzidos a estruturas de repetição como: "enquanto", "repita até", etc.

Sexto Dia Finalmente, a parte que exige maior capacidade de abstração dos alunos é deixada para o último dia de aula. Aqui os alunos desenvolverão habilidades para identificar trechos do programa que podem ser incorporados em funções. Então, ao invés de digitar o mesmo conjunto de comandos, agora os alunos poderão invocar suas próprias funções.

Semelhante à oficina básica, há uma alternativa ao formato intensivo de seis dias. No caso, são necessárias 12 aulas de duas hora cada, duas vezes na semana.

\section{Materiais e Métodos}

O plano de ensino, conforme apresentado na Seção 2, foi experimentado em duas escolas da rede pública estadual de ensino, ambas com oferta de ensino médio e fundamental maior (do sexto ao nono ano). Uma das escolas selecionada possui IDEB acima da média e está localizada na periferia da cidade, um bairro pobre, com elevado índice de violência. Ao longo do texto, esta escola será referenciada como Escola A. Enquanto que a segunda escola possui IDEB abaixo da média do estado e está localizada em área nobre, no centro da cidade, bem assistida de serviços e transporte. Esta segunda escola será referenciada no texto como Escola B.

Os exercícios e desafios da oficina foram planejados para alunos do sétimo ou oitavo anos do ensino fundamental. Além disso, em razão das diferentes realidades de gestão escolar e disponibilidade dos professores que encontramos nas duas escolas, a oficina ou era realizada no contra-turno das aulas, caso da Escola B, ou era realizada no mesmo turno das aulas, caso da Escola A.

Nas duas escolas pudemos experimentar as oficinas duas vezes, sempre com turmas diferentes. A primeira oficina foi realizada no meio do segundo semestre letivo, tanto na Escola A, como na Escola B. Enquanto que a segunda oficina foi realizada no início do primeiro semestre letivo em ambas as escolas que trabalhamos. Além disso, a primeira oficina, em ambas as escolas, foi executada em um modelo fracionado, ou seja, com aulas duas vezes por semana, duas horas por dia; enquanto que a segunda oficina, em ambas as escolas, foi executada em um modelo intensivo, ou seja, duas semanas de aula, todos os dias da semana, quatro horas por dia.

A fim de avaliarmos os efeitos da inclusão do ensino de programação de computadores, selecionamos em cada escola um conjunto de alunos para a intervenção (participação das aulas de Logo) e um conjunto de controle (não participação das aulas de Logo). No caso da Escola A, na primeira oficina a turma de intervenção selecionada estava no oitavo ano da manhã, e possuía 31 alunos, dos quais apenas 14 permaneciam no laboratório durante as aulas; enquanto a turma de controle selecionada estava no oitavo ano da tarde, e possuía 21 alunos. Na segunda oficina realizada na Escola A a turma de intervenção selecionada estava no oitavo ano da manhã, e possuía 29 alunos; enquanto a turma de controle selecionada estava no oitavo ano da tarde, e possuía 36 alunos. 
Na Escola B o conjunto de alunos de intervenção e controle pertenciam a mesma turma. Isso foi possível porque as duas oficinas ocorreram no contra-turno das aulas, portanto a adesão dos alunos as oficinas dificilmente seria de toda a turma. Assim, na primeira oficina 41 alunos pertenciam a turma, destes 7 frequentaram as aulas de Logo e representam o conjunto de intervenção, enquanto 34 alunos não frequentaram as aulas (conjunto de controle). Na segunda oficina na Escola B, 39 alunos pertenciam a turma, destes 13 frequentaram as aulas de Logo e representam o conjunto de intervenção, enquanto os 26 alunos não frequentaram as aulas (conjunto de controle).

\section{Resultados e Discussões}

O cronograma executado das oficinas precisou ser alterado algumas vezes, por exemplo, por causa da realização de jogos internos, semana de avaliação, feriados religiosos e dias facultados, falta de merenda escolar, falta de água, falta de energia, e reuniões administrativas, que ocasionaram a liberação dos alunos ou suspensão das atividades na escola. Apresentamos na Seção 4.1 uma análise quantitativa dos efeitos da exposição dos alunos à programação de computadores no desempenho de matemática, e na Seção 4.2 discutimos qualitativamente as diferenças que observamos ao executar as oficinas no mesmo turno e no contra-turno.

\subsection{Desempenho dos Alunos na Disciplina de Matemática}

Tabela 2. Primeira Oficina. Desempenho em Matemática: Mesmo turno.

\begin{tabular}{l|l|l|l|l|l}
\hline Conjunto & Alunos & $\mu$ Av. 3 & $\mu$ Av. 4 & $\sigma^{2}$ Av. 3 & $\sigma^{2}$ Av. 4 \\
\hline \hline Alunos que cursaram & 14 & 6,75 & 6,71 & 2,64 & 5,84 \\
\hline Alunos que não cursaram & 17 & 5,88 & 5,26 & 4,39 & 2,69 \\
\hline Alunos da tarde & 21 & 5,69 & 7,10 & 14,24 & 6,62
\end{tabular}

Tabela 3. Segunda Oficina. Desempenho em Matemática: Mesmo turno.

\begin{tabular}{l|l|l|l|l|l}
\hline Conjunto & Alunos & $\mu$ Av. 1 & $\mu$ Av. 2 & $\sigma^{2}$ Av. 1 & $\sigma^{2}$ Av. 2 \\
\hline \hline Alunos que cursaram & 29 & 3,41 & 4,79 & 1,34 & 4,56 \\
\hline Alunos que não cursaram & 36 & 3,07 & 3,13 & 5,79 & 4,45 \\
\hline
\end{tabular}

Tabela 4. Primeira Oficina. Desempenho em Matemática: Contra-turno.

\begin{tabular}{l|l|l|l|l|l}
\hline Conjunto & Alunos & $\mu$ Av. 3 & $\mu$ Av. 4 & $\sigma^{2}$ Av. 3 & $\sigma^{2}$ Av. 4 \\
\hline \hline Alunos que cursaram & 7 & 4,43 & 7,21 & 2,04 & 3,82 \\
\hline Alunos que não cursaram & 34 & 3,32 & 6,88 & 2,12 & 2,85 \\
\hline
\end{tabular}

Apresentamos na Tabela 2 e na Tabela 3 o desempenho médio dos alunos do conjunto de intervenção e controle, da Escola A, na disciplina de matemática. Para tanto, comparamos a média do aluno antes da oficina e após a oficina. No caso da primeira oficina, que aconteceu no meio do segundo semestre letivo, a média da terceira avaliação representa o desempenho do aluno antes da oficina e a média da quarta avaliação representa o desempenho do aluno após a oficina. Para a segunda oficina, que aconteceu no 
Tabela 5. Segunda Oficina. Desempenho em Matemática: Contra-turno.

\begin{tabular}{l|l|l|l|l|l}
\hline Conjunto & Alunos & $\mu$ Av. 1 & $\mu$ Av. 2 & $\sigma^{2}$ Av. 1 & $\sigma^{2}$ Av. 2 \\
\hline \hline Alunos que cursaram & 13 & 6,73 & 5,96 & 1,19 & 4,56 \\
\hline Alunos que não cursaram & 26 & 5,35 & 5,44 & 2,94 & 3,67 \\
\hline
\end{tabular}

meio do primeiro semestre letivo, a média da primeira avaliação representa o desempenho do aluno antes da oficina e a média da segunda avaliação representa o desempenho do aluno após a oficina.

Como podemos observar nas Tabelas 2 e 3, o desempenho médio dos alunos que frequentaram as aulas de Logo já era superior antes da intervenção. Especificamente no caso da primeira oficina, observamos: primeiro, a turma de intervenção parecia duas subturmas, uma dos alunos "interessados" e que frequentaram as aulas de Logo, e outra dos alunos "desinteressados" e que não optavam por sair do laboratório durante as aulas de Logo; segundo, a turma de controle melhorou bastante o desempenho, fato que pode estar associado a outras variáveis que não foram observadas neste estudo; terceiro, as diferenças entre as médias não era estatisticamente significativa. Enquanto que especificamente para a segunda oficina, observamos que a diferença de desempenho na média da segunda avaliação entre as turmas de intervenção e controle é estatisticamente significante (p-value $=0,002794$, para o Teste T, 95\% de confiança).

Nas Tabelas 4 e 5, apresentamos o desempenho médio dos alunos do conjunto de intervenção e controle, da Escola $\mathrm{B}$, na disciplina de matemática. Nesta escola também observamos que o desempenho dos alunos que frequentaram as aulas de Logo já era superior antes da intervenção e permaneceu superior após a intervenção. No caso da Escola B essa diferença de desempenho era previsível, pois acreditamos que os alunos que se voluntariaram a assistir aulas extra-curricular em um turno diferente de suas aulas são mais motivados e comprometidos com a sua formação - ao menos estão inseridos em um contexto familiar que estimula essa participação em atividades extra-classe. Por exemplo, observamos que no caso da segunda oficina, a diferença de desempenho na disciplina de matemática, entre os alunos que cursaram as aulas e os que não cursaram as aulas, na primeira avaliação, antes mesmo da oficina ser executada, era estatisticamente significativa ( -value $=0,007716$, para o Teste T, 95\% de confiança).

Quando analisamos, de forma pareada, apenas o grupo de alunos que assistiu as aulas de Logo, vale destacar que: na Escola A, na segunda oficina, o desempenho médio dos alunos aumentou de 3,41, na primeira avaliação, para 4,79, na segunda avaliação, uma diferença estatisticamente significativa ( $\mathrm{p}$-value $=0,0004376$, para o Teste T, $95 \%$ de confiança); na Escola B, na primeira oficina, o desempenho médio dos alunos aumentou de 4,43, na terceira avaliação, para 7,21, na quarta avaliação, uma diferença estatisticamente significativa ( $p$-value $=0,0102$, para o Teste $\mathrm{T}, 95 \%$ de confiança).

\subsection{Mesmo Turno e Contra-turno}

A experiência relatada neste trabalho nos permite destacar alguns pontos em relação à forma de oferta das oficinas no que diz respeito ao turno. Essas observações são importantes para o planejamento e execução de oficinas semelhantes no futuro.

Quando as oficinas são no mesmo turno, porque a computação não faz parte do 
currículo das escolas, os professores são convidados a ceder seus horários de aula. Ao mesmo tempo, a escola precisa planejar o uso do espaço do laboratório exclusivamente para a oficina, fato que pode comprometer o planejamento da escola como um todo. Assim, ou a oficina terá interrupções em função da demanda da escola, ou a escola precisará cessar sua demanda, oriunda de outras turmas, para que a oficina não sofra interrupções.

Ademais, os monitores das oficinas relataram maior dispersão durante as aulas e as atividades das oficinas realizadas no mesmo turno das aulas. Essas aulas contaram com a presença de todos os alunos matriculados, portanto, o laboratório normalmente ficava lotado. Acrescentando-se o problema do número limitado de computadores instalados e funcionando, observamos que a relação alunos por computador aumenta - em alguns momentos a relação alunos/computadores chegava a cinco. Por exemplo, eles identificaram que apenas $60 \%$ dos alunos demonstraram dedicação e interesse durante as oficinas realizadas no mesmo turno das aulas.

Quando as oficinas não são no mesmo turno das aulas, apesar de também concorrermos com o uso do laboratório, não precisamos da cessão de aulas dos professores. Por outro lado, necessitávamos da autorização dos responsáveis para que os alunos frequentassem a escola em um horário diferente das aulas.

Ademais, observamos uma adesão muito limitada dos alunos às aulas no contraturno. Por exemplo, menos da metade dos alunos se inscreveram nas oficinas (aproximadamente $38 \%$ de adesão), e destes, menos da metade concluíram as oficinas. Há várias explicações para a baixa adesão, porém as que observamos como mais latentes foram: a falta de recursos financeiros para o deslocamento à escola no contra-turno e a concorrência com outros programas de ensino ofertados na escola.

\section{Considerações Finais}

Este trabalho relata o primeiro contato, e os efeitos dessa experiência, que alunos do ensino fundamental de duas escolas públicas tiveram com a programação de computadores, com a linguagem Logo. Nesta experiência observamos a satisfação pessoal dos aprendizes ao terem contato pela primeira vez com o ambiente lúdico de programação. Por exemplo, através das observações e registros dos bolsistas, foi possível identificar que os alunos participantes das oficinas demonstraram motivação com a programação de computadores.

Notamos que em alguns cenários o desempenho dos alunos que assistiram as aulas de Logo melhorou ou foi superior ao desempenho daqueles alunos que não assistiram as aulas. Porém, esses resultados não são suficientemente conclusivos para generalizarmos ou indicarmos uma relação causal entre o contato com a programação de computadores e o desempenho na matemática, mas encoraja os autores a aprofundar a investigação dos efeitos deste contato.

Entre os diferentes modelos de execução da Oficina de Logo que experimentamos, observamos que é preferível que a mesma seja executada no mesmo turno das aulas e em modo intensivo. Além disso, considerando nosso desejo de ver o conteúdo de computação contemplado no currículo da educação básica, destacamos que os resultados de nossa experiência apresentados neste trabalho são animadores. Portanto, o plano de aula fracionado é uma alternativa viável para contemplar as aulas de Linguagem Logo. 


\section{Agradecimentos}

Os autores agradecem: (i) ao PIBID/CAPES/MEC; (ii) aos professores supervisores nas escolas; e (iii) aos diretores das escolas.

\section{Referências}

Alcoba, S. d. A. C. (2003). Logo, design e inclusão escolar. In Anais do Workshop de Informática na Escola, pages 266-274.

Barcelos, T. S. e Silveira, I. F. (2012). Pensamento computacional e educação matemática: Relações para o ensino de computação na educação básica. In XX Workshop sobre Educação em Computação, Curitiba, PR.

Bezerra, F. e Dias, K. (2014). Programação de computadores no ensino fundamental : Experiências com logo e scratch em escola pública. In XXII Workshop sobre Educação em Informática, pages 1515 - 1524, Brasília, DF. SBC.

Costa, T., Batista, A., Maia, M., Almeida, L., e Farias, A. (2012). Trabalhando fundamentos de computação no nível fundamental: experiência de licenciandos em computação da universidade federal da paraíba. In XX Workshop sobre Educação em Computação, Curitiba, PR.

de França, R. S., da Silva, W. C., e do Amaral, H. J. C. (2012). Ensino de ciência da computação na educação básica: Experiências, desafios e possibilidades. In $X X$ Workshop sobre Educação em Computação, Curitiba, PR.

Fincher, S., Cooper, S., Kölling, M., e Maloney, J. (2010). Comparing alice, greenfoot \& scratch. In Proceedings of the 41st ACM Technical Symposium on Computer Science Education, SIGCSE '10, pages 192-193, New York, NY, USA. ACM.

Freitas, R. C. d. O. e Santos, C. F. (2005). Trabalhando fractais no logo: uma experiência no ensino fundamental. In Anais do Workshop de Informática na Escola.

Friedrich, R. V., dos Santos, D. S., Keller, R. d. S., Puntel, M. D., e Biasoli, D. (2012). Proposta metodológica para a inserção ao ensino de lógica de programação com logo e lego mindstorms. In Anais do Simpósio Brasileiro de Informática na Educação.

Gregolin, V. R. (2009). Linguagem logo: Explorando conceitos matemáticos. Revista Tecnologias na Educação, 1(1):s/p.

Papert, S. (1993). Mindstorms: children, computers and powerful ideas. BasicBooks.

Pardamean, B., Evelin, E., e Honni, H. (2011). The effect of logo programming language for creativity and problem solving. In Proceedings of the 10th WSEAS International Conference on E-Activities, E-ACTIVITIES'11, pages 151-156, Stevens Point, Wisconsin, USA.

Torezani, C., Chagas, L. B. d. C., e Tavares, O. d. L. (2013). Newprog-um ambiente online para crianças aprenderem programação de computadores. In Anais do Workshop de Informática na Escola. 